\title{
Alterstice
}

Revue internationale de la recherche interculturelle

International Journal of Intercultural Research

Revista International de la Investigacion Intercultural

\section{Stratégies identitaires et stratégies d'acculturation : deux modèles complémentaires}

\section{Azzam Amin}

Volume 2, numéro 2, 2012

URI : https://id.erudit.org/iderudit/1077569ar

DOI : https://doi.org/10.7202/1077569ar

Aller au sommaire du numéro

Éditeur(s)

Alterstice

ISSN

1923-919X (numérique)

Découvrir la revue

Citer cet article

Amin, A. (2012). Stratégies identitaires et stratégies d'acculturation : deux modèles complémentaires. Alterstice, 2(2), 103-116.

https://doi.org/10.7202/1077569ar

\section{Résumé de l'article}

Dans cet article, nous proposons de mener une réflexion épistémologique, théorique et méthodologique à partir d'une confrontation entre le modèle des stratégies identitaires de Camilleri et celui des stratégies d'acculturation de Berry. La comparaison et la discussion de ces deux modèles et de leur contribution à l'étude des dynamiques interculturelles en psychologie montrent la possibilité et la nécessité de les articuler afin de tenir compte de la complexité des phénomènes interculturels. L'articulation de ces deux approches différentes mais complémentaires est basée sur le concept de l'identité dans ses multiples dimensions personnelle et sociale. Ella a comme objectif d'ouvrir de nouvelles perspectives de recherche sur les contacts de cultures. 


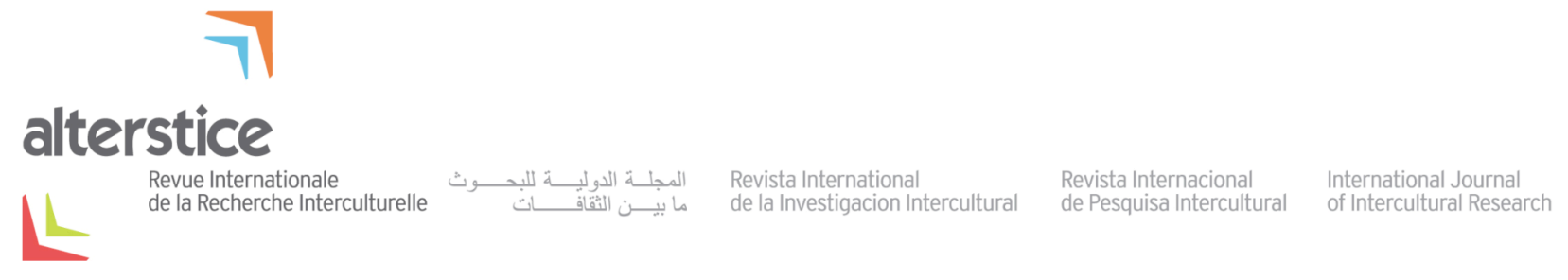

ARTICLE HORS THÈME

\section{Stratégies identitaires et stratégies d'acculturation : deux modèles complémentaires}

Azzam Amin ${ }^{1,2}$

\section{Résumé}

Dans cet article, nous proposons de mener une réflexion épistémologique, théorique et méthodologique à partir d'une confrontation entre le modèle des stratégies identitaires de Camilleri et celui des stratégies d'acculturation de Berry. La comparaison et la discussion de ces deux modèles et de leur contribution à l'étude des dynamiques interculturelles en psychologie montrent la possibilité et la nécessité de les articuler afin de tenir compte de la complexité des phénomènes interculturels. L'articulation de ces deux approches différentes mais complémentaires est basée sur le concept de l'identité dans ses multiples dimensions personnelle et sociale. Ella a comme objectif d'ouvrir de nouvelles perspectives de recherche sur les contacts de cultures.

\section{Rattachement de l'auteur}

${ }^{1}$ Université Catholique de Lyon, Lyon, France; ${ }^{2}$ Groupe de recherche en psychologie sociale (GRePS)

\section{Correspondance}

aamin@univ-catholyon.fr

\section{Mots clés}

psychologie interculturelle; stratégies identitaires; acculturation; modèle intégrateur

\section{Pour citer cet article :}

Amin, A. (2012). Stratégies identitaires et stratégies d'acculturation : deux modèles complémentaires. Alterstice, 2(2), 103-116. 


\section{Introduction}

Dans le contexte actuel où les individus et les groupes se déplacent de manière intense dans le monde, les contacts de cultures sont devenus un phénomène structurel de nos sociétés (Camilleri, 1996a). Les situations de rencontres interculturelles, interpersonnelles et intergroupes sont omniprésentes au point que l'on peut avancer, à l'instar de Troadec (2001), que le contact des cultures a des effets sur tous et que nul n'est enfermé dans une position statique.

Différents modèles en psychologie ont été élaborés pour étudier la dynamique interculturelle ainsi que ses répercussions sur l'identité individuelle et sociale. Dans le domaine de la recherche francophone, le modèle des stratégies identitaires, principalement représenté par les travaux de Camilleri (1989, 1990, 1992, 1996a, 1996b) incarne l'une des contributions majeures. Concernant la recherche anglophone, les études ont été centrées sur les processus d'acculturation et leur impact sur les individus et les sociétés. Le modèle des stratégies d'acculturation de Berry (1989, 1990, 2000; voir aussi Berry et Sabatier, 2010) est le plus représentatif dans ce domaine. Ces deux modèles ont eu une grande influence sur la recherche en psychologie interculturelle (Sabatier, 2001; Sabatier et Boutry, 2006). Bien qu'ils concernent le même champ de recherche, à savoir celui des processus liés aux contacts de cultures et aux changements qui en découlent, ces deux modèles sont restés longtemps parallèles. Dans un livre publié en hommage à Camilleri, Dasen et Ogay (2000) soulignent la nécessité d'articuler les travaux sur les stratégies identitaires avec ceux sur les stratégies d'acculturation.

L'objectif de cet article est de débattre de la pertinence de ces deux modèles et de leur contribution à l'étude des dynamiques interculturelles, et de les confronter dans leurs approches épistémologiques, théoriques et méthodologiques. En nous appuyant sur l'analyse de Dasen et Ogay, cette confrontation prendra la forme de réflexions épistémologiques et permettra de proposer une articulation entre ces deux approches dans l'objectif d'ouvrir un nouvel ensemble de pistes de recherches sur les contacts de cultures.

\section{Le modèle des stratégies identitaires de Camilleri}

L'origine de la notion de stratégie identitaire prend racine dans la théorie de l'identité sociale de Tajfel et Turner $(1979,1986)$. Ces auteurs partent du postulat selon lequel tout individu est à la recherche d'une estime de soi positive (identité positive) et que son appartenance à des groupes sociaux en dépend. Quand cette identité est dévalorisée ou remise en question, l'individu recourt à des stratégies identitaires pour la restaurer. Camilleri (1990) reprend cette idée comme base de sa théorie des stratégies identitaires en situation interculturelle, telle la situation des populations migrantes. Dasen et Ogay (2000) ont proposé le tableau ci-contre (tableau 1) pour illustrer ces stratégies telles qu'elles ont été formulées par Camilleri.

D'après Camilleri (1990), le migrant vit le contact entre sa culture d'origine et celle de la société d'accueil comme un conflit, un morcellement culturel, et subit ainsi une pression psychologique se répercutant sur son système identitaire. Cette répercussion se traduit sous forme de deux bouleversements.

Tout d'abord, l'individu migrant connaît des atteintes à l'unité de sens, où son équilibre est mis à mal car les valeurs "traditionnelles» auxquelles il s'identifie, c'est-à-dire les valeurs définissant son identité d'origine, ne lui permettent plus de s'accorder avec son nouvel environnement "moderne ». La cohérence entre ce que Camilleri appelle la fonction ontologique de l'identité (relative à son enculturation) et la fonction pragmatique (relative à la nécessité de s'adapter à l'environnement) est ébranlée. Pour restaurer cette cohérence, l'individu a recours à trois types de stratégies: les stratégies d'évitement des conflits identitaires par la cohérence simple, les stratégies d'évitement des conflits par la cohérence complexe et les stratégies de modération des conflits (Camilleri, 1989 et 1990). 
Tableau 1 : Typologie des stratégies identitaires selon Camilleri, rapportée par Dasen et Ogay (2000, p. 58).

\begin{tabular}{|c|c|c|c|}
\hline \multicolumn{4}{|c|}{ L'individu en situation d'acculturation rencontre dévalorisation et destruction } \\
\hline \multicolumn{2}{|r|}{$\begin{array}{l}\text { Stratégies pour rétablir le sentiment } \\
\text { valeur du soi }\end{array}$} & \multicolumn{2}{|c|}{$\begin{array}{l}\text { Stratégies pour rétablir une unité de sens (cohérence } \\
\text { entre la fonction ontologique et la fonction pragmatique) }\end{array}$} \\
\hline \multirow{3}{*}{ 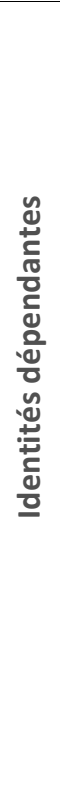 } & $\begin{array}{l}\text { Identité négative : } \\
\text { intériorisation du jugement dépréciatif. }\end{array}$ & \multirow{3}{*}{$\begin{array}{l}\text { Cohérence } \\
\text { simple : } \\
\text { résolution de la } \\
\text { contradiction } \\
\text { par la } \\
\text { suppression de } \\
\text { l'un de ses } \\
\text { termes }\end{array}$} & $\begin{array}{l}\text { Survalorisation de la fonction } \\
\text { ontologique : } \\
\text { investissement plus ou moins exclusif } \\
\text { dans le système d'origine } \\
\text { (fondamentaliste, conservateur total). }\end{array}$ \\
\hline & $\begin{array}{l}\text { Identité négative déplacée : évacuation } \\
\text { de l'identité négative en s'assimilant au } \\
\text { favorisé et en transférant l'injonction } \\
\text { dévalorisante sur les autres membres } \\
\text { de son groupe d'origine. }\end{array}$ & & $\begin{array}{l}\text { Valorisation dominante de la fonction } \\
\text { pragmatique mais conservation d'un } \\
\text { minimum ontologique : } \\
\text { alternance conjoncturelle des codes } \\
\text { (opportuniste limité). }\end{array}$ \\
\hline & $\begin{array}{l}\text { Identité par distinction : } \\
\text { prise de conscience de sa singularité } \\
\text { mais non intériorisation de la } \\
\text { dévalorisation, évitée par la prise de } \\
\text { distance. }\end{array}$ & & $\begin{array}{l}\text { Survalorisation de la fonction } \\
\text { pragmatique : } \\
\text { investissement plus ou moins exclusif } \\
\text { dans le système d'accueil, primauté de } \\
\text { la volonté d'adaptation (opportuniste } \\
\text { complet). }\end{array}$ \\
\hline \multirow{3}{*}{ 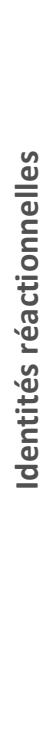 } & $\begin{array}{l}\text { Identité de défense: } \\
\text { l'identité comme refus, comme bouclier } \\
\text { pour se protéger des autres. }\end{array}$ & \multirow{2}{*}{$\begin{array}{l}\text { Cohérence } \\
\text { complexe : } \\
\text { tenir compte de } \\
\text { tous les } \\
\text { éléments en } \\
\text { opposition }\end{array}$} & $\begin{array}{l}\text { Bricolages identitaires: } \\
\text { résolution de la contradiction pour soi } \\
\text { et non en soi, logique effective et non } \\
\text { rationnelle. }\end{array}$ \\
\hline & $\begin{array}{l}\text { Identité polémique : } \\
\text { sur-affirmation des caractères } \\
\text { stigmatisés, en opposition } \\
\text { généralement agressive contre le } \\
\text { dominant. }\end{array}$ & & $\begin{array}{l}\text { Logique rationnelle: } \\
\text { réappropriation, dissociation, } \\
\text { articulation organique des contraires, } \\
\text { suspension de l'application de la valeur, } \\
\text { valorisation de l'esprit aux dépens de la } \\
\text { lettre. }\end{array}$ \\
\hline & $\begin{array}{l}\text { Identité de principe } c .-\grave{a}-d \text {. } \\
\text { volontariste: } \\
\text { conduite paradoxale de revendication } \\
\text { d'appartenance au groupe d'origine, } \\
\text { alors que rejet de ses valeurs dans les } \\
\text { actes. }\end{array}$ & $\begin{array}{l}\text { Stratégies de } \\
\text { modération des } \\
\text { conflits }\end{array}$ & $\begin{array}{l}\text { Stratégies problématiques ne } \\
\text { permettant pas d'éviter le conflit : } \\
\text { pondération différentielle des valeurs } \\
\text { en opposition, } \\
\text { limitation de l'élément perçu comme } \\
\text { pénible, alternance systématisée des } \\
\text { codes. }\end{array}$ \\
\hline
\end{tabular}

La cohérence simple correspond à une alternance des codes à la disposition de l'individu. Ainsi, selon la situation, le migrant se réfère soit à sa préoccupation ontologique, c'est-à-dire qu'il s'investit entièrement dans le système originel (bien que cette stratégie soit coûteuse car un minimum de pragmatisme est nécessaire), soit à la préoccupation pragmatique mettant en avant la volonté d'adaptation à l'environnement. 
Concernant les stratégies d'évitement des conflits par la cohérence complexe, l'individu migrant combine les deux préoccupations (pragmatique et ontologique). Camilleri $(1989,1996 a)$ distingue deux sortes de mise en lien dans ce type de stratégie : le sujet migrant peut, en effet, mettre en lien les deux préoccupations sans se référer à la logique rationnelle, c'est-à-dire qu'il interprète les codes de façon égocentrique en manipulant leur sens de sorte que la cohérence entre les deux préoccupations soit maintenue. Il peut à l'inverse mettre en lien les deux préoccupations selon la logique rationnelle en avançant des arguments irréfutables qui seraient acceptables dans les deux cultures en contact.

Enfin, les stratégies de modération des conflits de codes correspondent à la diminution du conflit qui est parvenu à s'installer. L'individu peut pour cela investir inégalement les valeurs en contradiction, ce que l'on appelle la pondération différentielle des valeurs en opposition. Il peut aussi limiter la perception de l'élément ressenti comme pénible, ou bien alterner les codes en compensant une activité culpabilisante au vu des valeurs d'origine par un fort investissement dans une autre activité qui y est rattachée.

Le deuxième bouleversement auquel le sujet migrant doit faire face concerne les atteintes à l'image de soi résultant de l'influence de la situation de dominé du migrant dans la société d'accueil. D’après Camilleri (1990), cette situation a pour conséquence la prescription par le dominant à l'individu dominé d'un ensemble de caractéristiques connotées le plus souvent négativement : jugements de valeur et stéréotypes racistes attribués au groupe d'appartenance et aux rôles sociaux. La structure identitaire s'élabore alors en fonction de ces prescriptions qui ne tiennent pas compte de la réalité de l'individu.

Face à cette atteinte à l'auto-attribution de la valeur, le sujet peut user de plusieurs stratégies moins complexes que celles concernant la préservation de la cohérence, mais cela ne signifie pas qu'elles n'ont pas leur importance pour lui sur le plan pratique. Les migrants ont recours à deux types de stratégies :

D'une part, il y a les "identités dépendantes " qui contribuent à maintenir l'identité du sujet migrant sous la dépendance des injonctions identitaires de l'environnement. Dans cette modalité, les migrants intériorisent l'image négative prescrite et sont donc portés à signifier leur réalité et leurs valeurs selon les injonctions de la société, ce que Camilleri (1990) appelle l'« identité négative ». D'autres essaient d'évacuer les jugements dépréciatifs de la part de la société d'accueil en s'immergeant totalement dans l'ensemble socioculturel. Ils recourent alors à l'assimilation et déplacent l'image négative vers les autres membres de la communauté d'origine: il s'agit de l'« identité négative déplacée ». Le sujet peut également se distinguer en prenant conscience de sa singularité sans intérioriser la dévalorisation, l'écart mis entre lui et son environnement diminuant l'impact des prescriptions négatives. Dans ce cas, on parlera d'« identité par distinction ».

D’autre part, on trouve les «identités réactionnelles » qui expriment une volonté du sujet de s'émanciper de l'image négative prescrite. En effet, afin d'éviter la dévalorisation, certains sujets vont revendiquer leur origine, soit en l'utilisant comme bouclier pour se protéger des autres, perçus comme dépréciateurs (identité défense), soit en sur-affirmant les caractères stigmatisés afin d'échapper ainsi aux sentiments dévalorisants (identité polémique). Camilleri (1996a) ajoute que, pour échapper à la dévalorisation, certains sujets revendiquent leur appartenance au groupe d'origine, alors qu'ils rejettent ses valeurs dans leurs actes (identité de principe).

Comme nous pouvons le remarquer dans le tableau 1, la théorie des stratégies identitaires est particulièrement diversifiée et riche. Exposer toutes les stratégies identitaires étudiées est un exercice ardu car celles-ci présentent de nombreuses variantes selon les auteurs et parfois chez un même auteur (Amin, 2007).

\section{Le modèle des stratégies d'acculturation de Berry}

Selon Redfield, Linton et Herskovits (1936), l'acculturation renvoie à « l'ensemble des phénomènes résultant d'un contact continu et direct entre groupes d'individus appartenant à différentes cultures, et aboutissant à des transformations qui affectent les modèles culturels originaux de l'un ou des deux groupes " (p. 149, notre traduction). Bien qu'en principe les changements soient mutuels, il existe généralement un groupe qui domine, dit 
"groupe dominant » qui jouit d'une influence culturelle plus forte que l'autre groupe, dit "groupe dominé ", souvent composé de migrants et de leurs descendants (Berry et Sam, 1997).

L'individu dans une situation d'acculturation recourt à des stratégies pour s'adapter à la nouvelle société. Ces stratégies comprennent : le niveau attitudinal, ou attitudes d'acculturation, relatif au positionnement de l'individu entre les deux cultures en contact : le niveau comportemental, qui concerne les changements de comportements individuels et de conduites sociales dans la nouvelle société (Sabatier et Berry, 1994). Certaines situations d'acculturation représentent de nombreuses sources de difficultés et, si cela est problématique, on observe un état de stress, soit le stress d'acculturation (Berry, 1980 et 2000; Berry et Sabatier, 2010; Berry et Sam, 1997).

Le choix des stratégies d'acculturation se fait d'abord en fonction du positionnement de l'individu entre sa culture d'origine et celle de la société d'accueil. Selon Berry (2000), le niveau attitudinal détermine le niveau comportemental et le stress d'acculturation. L'individu se positionne entre les cultures en contact selon deux dimensions : la première concerne la volonté d'avoir des contacts et des participations avec la société d'accueil et d'adopter ses valeurs. La deuxième est liée au maintien de la culture d'origine, de l'identité culturelle et de ses coutumes au sein de la société d'accueil. Ces dimensions se formulent en deux questions que se pose tout individu migrant, d'origine immigrée ou faisant partie d'une minorité culturelle :

- Faut-il maintenir sa culture et son identité d'origine?

- Faut-il avoir des contacts avec les membres de la société d'accueil et participer à la vie sociale de cette société?

Le croisement des réponses « oui » ou " non » à ces questions permet de classer le choix de l'individu en quatre stratégies d'acculturation, représentées dans le tableau 2.

Tableau 2 : Les stratégies d'acculturation selon Berry et Sam (1997, p. 296).

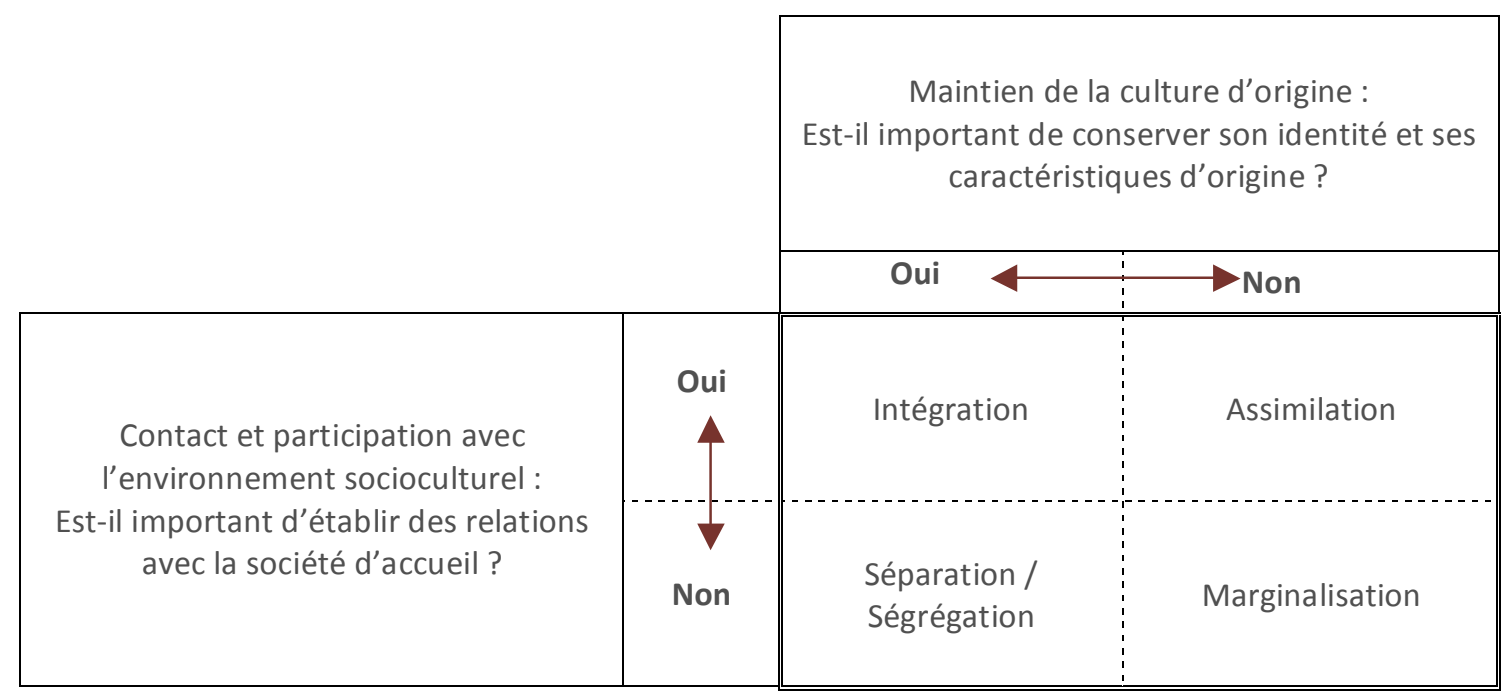

Dans l'intégration, l'individu veut à la fois maintenir sa culture et son identité d'origine et avoir des contacts avec la société d'accueil. II participe ainsi à la vie sociale dans la société d'accueil tout en conservant sa culture d'origine. Dans ce cas, il existe plusieurs groupes ethniques distincts, coopérant tous au sein du système social général; le modèle multiculturel canadien en est un exemple (Guimond, 2010). L'individu peut alors mélanger les valeurs de sa culture d'origine et celles de la culture de la société d'accueil. 
Avec l'assimilation, l'individu abandonne son identité et sa culture d'origine et cherche à établir des relations avec la société d'accueil. Il adopte alors la culture de la société d'accueil au détriment de sa culture d'origine. Cela peut conduire à l'absorption du groupe d'acculturation par le groupe dominant (Sabatier et Berry, 1994).

Par la séparation, l'individu cherche à conserver son identité et sa culture d'origine, tout en évitant volontairement des interactions ou des relations avec la société d'accueil. Si cette absence de relation avec la société d'accueil est imposée par cette société elle-même, on parlera davantage de "ségrégation ". Comme Berry et Sabatier (2010) le font remarquer, c'est l'origine du choix (libre ou imposé) qui détermine ici la stratégie (séparation ou ségrégation).

Enfin, la marginalisation conduit l'individu à perdre son identité culturelle sans pouvoir établir des interactions ou des relations avec la société d'accueil. Plusieurs chercheurs parlent dans ce cas d'identité aliénée (Sabatier et Berry, 1994). Cette situation est difficile à cerner avec précision et s'accompagne de confusion identitaire collective et individuelle, d'angoisse et de stress. Elle relève plutôt de situations pathologiques et pourrait être le résultat de discrimination et d'exclusion à l'égard de l'individu migrant (Berry, 1980 et 2000).

\section{Interrogations et confrontations épistémologiques}

Notre lecture de ces deux modèles laisse apparaître plusieurs interrogations concernant leurs approches des contacts de cultures, des processus psychologiques individuels sous-jacents et de leur rôle dans les phénomènes interculturels, ainsi que leurs limites méthodologiques.

\section{Approches différentes des contacts de cultures}

Notre première interrogation concerne la différence conceptuelle entre ces deux approches distinctes des phénomènes de contacts de cultures, l'une s'inscrivant dans la tradition francophone de la recherche interculturelle francophone, et l'autre dans la tradition anglophone. Le premier point de divergence concerne le concept d'acculturation. Critiqué par certains chercheurs francophones (Sabatier et Boutry, 2006), le concept d'acculturation serait imprégné d'ethnocentrisme et difficilement dissociable du champ de l'anthropologie culturelle pour être utilisé dans un autre champ des sciences humaines. En effet, l'anthropologie culturelle est liée à l'époque du colonialisme et de fait, le terme d'acculturation est marqué par les idéologies et les conceptions asymétriques des relations entre cultures véhiculées à l'époque. Autrement dit, l'acculturation a une connotation unilatérale. Bien que Redfield, Linton et Herskovits (1936) aient mis l'accent sur les changements mutuels, ces relations sont souvent asymétriques: une culture dominante qui impose son modèle à une culture dominée qui doit s'y conformer (Vasquez, 1984). La notion d'acculturation impliquerait également un ordre temporel, diachronique, c'est-à-dire qu'il y aurait un changement de contexte dans le temps, une culture (considérée comme deuxième) se présenterait dans une autre considérée comme première (Clanet, 1990). Ce concept ne peut donc être appliqué qu'à des individus ayant quitté leur pays d'origine où ils ont acquis la culture de leur groupe ethnique. Cela n'est pas du tout le cas des jeunes issus de l'immigration, nés et socialisés dans les deux systèmes culturels (Amin, 2007 ; Manço, 2006).

Toutefois, il faut souligner que ce n'est pas le concept lui-même mais son utilisation dans certaines études qui ont eu tendance à développer une conception linéaire et statique de l'acculturation en rappelant que la définition originelle suppose une interdépendance des cultures (Belkaïd et Guerraoui, 2003).

Certains chercheurs en France préfèrent utiliser le terme d'interculturation (Clanet, 1990; Denoux, 1994 et 1994 1995; Guerraoui, 1992). Ce dernier est lié à la synchronie, à l'alternance et à la simultanéité des changements culturels. Le sujet se structure alors dans une double référence symbolique hétérogène et irréductible à l'un des deux pôles culturels.

La deuxième différence entre le modèle de Camilleri et celui de Berry concerne la place du " conflit culturel » dans les processus identitaires des sujets migrants. En effet, dans le premier, le conflit culturel est considéré comme un postulat pour expliquer les mécanismes identitaires mis en œuvre par des sujets pris individuellement, ce qui n'est pas le cas dans le modèle de Berry. Il est vrai que le conflit de valeurs existe et influence sans doute les individus migrants ou issus de l'immigration, mais son rôle nous semble surévalué dans les travaux de Camilleri (1990) et il 
pourrait laisser entendre qu'il existe une sorte d'incompatibilité culturelle qui détermine finalement ces processus. Autrement dit, plus la culture de la société d'accueil et la culture d'origine des migrants sont proches, plus l'intégration est rapide et facile (Dasen et Ogay, 2000). Il s'agit d'une analyse "manichéenne qui ne fait que renforcer les stéréotypes » (p. 66), qui ne fait qu'accentuer les visions xénophobes des relations interculturelles. Bien entendu, Camilleri et tous ceux qui ont travaillé avec lui sont loin de ces représentations racistes, mais il y a un risque d'effet pervers dans leurs écrits.

La notion de conflit culturel et ses présupposés, souvent définis en termes de perte de repères et de confusions identificatoires chez les migrants et leurs enfants, impliquent une hiérarchisation linéaire et évolutionniste entre la culture du pays d'origine perçue comme traditionnelle (archaïque) et la culture de la société d'accueil présentée comme moderne (évoluée). À partir de là, le fait que le migrant tienne à sa culture d'origine pourrait être interprété comme une résistance à la société d'accueil, voire une menace. Au contraire, accepter les valeurs présumées modernes de cette dernière va de pair avec l'intégration. De plus, la dualité tradition/modernité contient, en elle-même, un jugement de valeur. Lorsque l'on associe la tradition à une culture d'origine et la modernité à celle de la société d'accueil, le réductionnisme devient évident (Balkaïd, 2000). La notion de conflit culturel ainsi que la dualité traditionnel/moderne sont basées sur un jugement comparatif asymétrique. De fait, nous avons l'impression que plus la culture du sujet migrant est jugée différente de celle de la société d'accueil, plus elle est jugée traditionnelle, et que cette opposition systématique entre les valeurs peut engendrer, encore une fois, des dérives.

Sans pour autant ignorer cette conflictualité et le rôle que la différence culturelle peut jouer sur le plan individuel et collectif dans une situation de contacts interculturels, il semble néanmoins risqué de les mettre à la base d'un modèle d'analyse des problèmes d'intégration. C'est pour cette raison que Camilleri $(1989,1990)$ invite à ne pas confondre différence et hiérarchie. Ce n'est pas la différence en soi qui doit être interrogée, mais le sens donné à cette dernière. En effet, la différence culturelle a longtemps été utilisée par un certain nombre de courants idéologiques pour justifier les politiques assimilationnistes de l'immigration (Guimond, 2010). À l'inverse, dans ses analyses des processus d'acculturation, Berry $(1989,2000)$ évoque les rapports entre des groupes dominants et dominés et leurs rôles dans les mécanismes identitaires, ajoutant ainsi une dimension sociale à la dimension culturelle mise en avant par Camilleri $(1989,1990)$.

Enfin, selon ce dernier, la finalité des stratégies identitaires est de réduire la souffrance intrapsychique et de retrouver l'équilibre du fait de la dévalorisation des migrants par la société d'accueil et des rapports d'inégalité, de dépréciation, de rejet et de discrimination entretenus. Cette vision négative est constatée dans la plupart des travaux qui s'appuient sur la théorie des stratégies identitaires. Nous pensons notamment à ceux de MalewskaPeyre $(1989,1990)$. Ceci pourrait être vrai pour la société française qui pratique souvent une politique assimilationniste de l'immigration (Guimond, 2010). Cette dernière considère le multiculturalisme et I'hétérogénéité culturelle comme une menace envers l'unité nationale de la France (Clanet, 1990), mais en est-il de même pour les autres pays? Le conflit est-il inhérent aux contacts de cultures? « Tous les auteurs ne partagent pas cette vision pessimiste et rappellent que l'acculturation ne représente pas seulement un risque mais aussi une chance " (Dasen et Ogay, 2000, p. 66).

Au Canada, où les travaux de Berry ont débuté, l'idéologie politique est propice au pluralisme culturel, favorisant ainsi l'adaptation des institutions à la diversité ethnoculturelle et donnant lieu à une tonalité et une vision optimistes des contacts de cultures dans les recherches sur les stratégies d'acculturation (Berry, 2000; Berry et Sabatier, 2010; Sabatier et Berry, 1994).

\section{Le rôle des processus psychologiques individuels dans les phénomènes interculturels}

Partant d'une approche individualisante et volontariste des processus identitaires et de la gestion de la disparité culturelle, Camilleri $(1989,1990,1996 a)$ considère que l'individu est au centre des processus identitaires. II est acteur dans les négociations identitaires : il décide et choisit ses réponses face à cette disparité culturelle. Cette conceptualisation a permis, comme nous l'avons relevé plus haut, de concevoir une riche diversité dans les stratégies identitaires et les processus sous-jacents à l'acculturation, mais ignore toutefois les aspects contextuels

Alterstice - Revue Internationale de la Recherche Interculturelle, vol. 2, $n^{\circ} 2$ 
(sociaux, économiques, politiques...) qui influencent l'individu. Même si Camilleri évoque le rôle de la société dominante dans la dévalorisation de l'image de soi et dans le conflit culturel, ce dernier est un fait objectif résolu subjectivement car il implique des conflits intra-subjectifs. II semble contradictoire de considérer par avance l'existence d'un risque de conflit intra-subjectif, alors que la source de celui-ci est vue comme objective, extérieure au sujet concerné. Peut-on imputer la responsabilité de la gestion de la pluralité culturelle et de l'intégration au dominé (individu) plutôt qu'au système politique, social et économique? (Amin, 2007). Il semble dommage de voir une telle richesse théorique et scientifique amputée de ces aspects fondamentaux.

Berry parle de " choix » dans les stratégies d'acculturation adoptées, sans prendre en compte le conditionnement social, historique, idéologique et culturel, qui dépasse souvent l'individu, ainsi que les paramètres psychologiques inconscients qui s'imposent à lui. Peut-on alors considérer les relations interculturelles et leurs conséquences comme émanant d'un choix, soit dotées d'une intentionnalité (Amin, 2005; Andronikof-Sanglade et Franchi, 2001)? Pourtant, Berry lui-même (2000) a souligné l'importance des attitudes de la société d'accueil et de sa politique envers le groupe dominé dans l'étude de l'acculturation, ainsi que la nécessité de tenir compte de la complexité des variables qui entrent en jeu (Berry et Sam, 1997).

En réponse à cela, Bourhis, Barrette, El-Geledi, et Schmidt (2009), Bourhis et Bougie (1998), Bourhis, Moise, Perreault et Senecal (1997), Bourhis, Personnaz, Barrette et Personnaz (2002) ont proposé le modèle d'acculturation interactif (MAI) selon lequel les stratégies d'acculturation endossées par les membres de la société d'accueil ont un impact fort sur les modes d'acculturation des migrants ou des minorités culturelles. Ils rappellent aussi que les attitudes des membres de deux cultures en contact (minorité/majorité, dominée/dominante, immigrés/société d'accueil) sont influencées par les politiques d'immigration adoptées par le gouvernement au niveau national. Ce cadre théorique montre que les stratégies d'acculturation ne sont qu'un facteur parmi de nombreux autres (Dasen, 2001).

\section{Des limites méthodologiques}

Notre dernière interrogation porte sur l'aspect méthodologique et la pertinence d'une éventuelle généralisation du modèle des stratégies identitaires et celui des stratégies d'acculturation. Camilleri $(1990,1992)$ affirme à plusieurs reprises que sa théorie est généralisable "dans une mesure variable à d'autres sociétés et d'autres groupes d'immigrés» (1989, p.62). Pourtant, le modèle des stratégies identitaires a ses limites méthodologiques concernant cette question. En effet, la typologie proposée par Camilleri a été théorisée et élaborée essentiellement en France, et uniquement avec une population maghrébine, c'est-à-dire dans un contexte socio-politique de type assimilationniste et auprès d'une population ayant une relation historique et culturelle particulière avec la société d'accueil. Dans ce cas précis, il semble discutable de considérer ce modèle comme généralisable sinon universel (Dasen et Ogay, 2000). Si le chercheur veut « parvenir à une compréhension globale des principes (scientifiques) généraux qui président aux relations interculturelles, on ne doit pas se cantonner à l'étude d'un seul type de groupe, appartenant à une espèce de culture et établi dans un certain pays » (Berry, 2000, p. 91). II est regrettable que le modèle des stratégies identitaires n'ait pas été mis à l'épreuve sur d'autres populations et d'autres sociétés d'accueil, comme ont pu le faire Berry et ses collègues (Berry et Sam 1997, Berry, Phinney, Sam et Vedder, 2006). En effet, le modèle des stratégies d'acculturation a été testé dans différents pays (Australie, Canada, Inde, France...) et sur plusieurs groupes culturels (Italiens, Coréens, Maghrébins, Turcs...).

L'une des différences importantes entre la recherche francophone et la recherche anglophone se veut méthodologique. Dans la première, les études sont plutôt qualitatives. Elles se centrent davantage sur la description des aspects psychiques individuels liés aux phénomènes de contacts de cultures, et les données sont approfondies, diversifiées et cliniques. Dans la seconde, elles sont davantage quantitatives. Elles visent à expliquer et privilégient la recherche d'indices sur la dynamique globale des groupes culturels en contact, notamment comportementaux (Brégent, Mokounkolo et Pasquier, 2008; Sabatier et Boutry, 2006).

Toutefois, si la rigueur méthodologique du modèle des stratégies d'acculturation a permis à l'auteur d'exporter son modèle, sa simplicité l'a empêché de prendre en compte toute la complexité de l'être humain et l'a dirigé vers une schématisation et un cloisonnement des stratégies d'acculturation réellement développées. Bien que Berry et Sam 
(1997) aient pris en compte la dynamique de ces stratégies en rajoutant des flèches dans le schéma afin de souligner davantage qu'il y a un continuum de réponse entre oui et non (voir tableau 2), les liens entre ces stratégies restent ambiguës. En effet, si un même individu peut osciller d'une stratégie à l'autre tout au long de sa vie, il peut également se situer à un niveau plus ou moins intermédiaire entre elles en fonction de ses interactions avec le contexte dans lequel il se retrouve. Cette flexibilité et cette variabilité constituent une spécificité du modèle des stratégies identitaires. À ce propos, Camilleri affirme que la question des stratégies « est évoquée par J. W. Berry, mais sur un plan très général et sans entrer dans le détail analytique des chercheurs francophones » (1996b, p. 102).

\section{Vers une articulation de deux approches}

Nos questionnements critiques à l'égard du modèle de Camilleri et de celui de Berry n'ont pas comme objectif de remettre en question leur pertinence et leurs contributions à la recherche interculturelle. Bien au contraire, nous pensons qu'ils constituent un soubassement indispensable à la compréhension des conduites en situations interculturelles. Néanmoins, il semble pertinent de prendre un peu de recul et de trouver une manière originale de comprendre à la fois les besoins personnels d'un immigrant, comme celui de donner du sens, par exemple, dans la gestion d'une identité interculturelle complexe, tout en tenant compte de ses besoins sociaux et de l'exigence qu'il a à concilier les diverses demandes (pressions) provenant des différents groupes auxquels il appartient ou non.

Les modèles des stratégies identitaires et d'acculturation nous semblent complémentaires pour l'étude des contacts de cultures. Cette complémentarité se traduit par la présence conjointe des divergences et des ressemblances sur les aspects contextuels, méthodologiques et conceptuels; ressemblances que nous évoquons dans cette partie du texte.

Même si le concept d'acculturation a été pendant longtemps biaisé par le fait qu'historiquement il a été le fait d'auteurs issus de sociétés d'immigration (industrialisées), en considérant implicitement ou explicitement qu'il revenait aux « arrivants " (issus de sociétés non industrialisées) de s'adapter à la culture de la société d'accueil, il a évolué. Par conséquent, nous pouvons à l'instar de Bourhis et collab. (1997) proposer une vision interactive des phénomènes d'acculturation. Camilleri (cité par Kanouté, 2002), lors de la conférence d'ouverture du Vle congrès de I'ARIC (Association francophone pour la Recherche Inter Culturelle) en 1996 à Montréal, a souligné que les stratégies identitaires sont "impliquées » d'une certaine manière dans les stratégies d'acculturation. Ces deux modèles adoptent la notion de stratégie pour expliquer les conduites des individus dans les situations interculturelles. Elle se définit « comme des procédures mises en œuvre (de façon consciente ou inconsciente) par un acteur (individuel et collectif) pour atteindre une, ou des, finalités (définies explicitement ou se situant au niveau de l'inconscient), procédures élaborées en fonction de la situation d'interaction, c'est-à-dire en fonction des différentes déterminations (socio-historiques, culturelles, psychologiques) de cette situation » (Lipiansky, TaboadaLéonetti et Vasquez, 1990, p. 24). Le choix d'usage de cette notion réside dans l'idée que les individus sont des sujets acteurs capables d'agir sur leur identité, « cette conception est une conséquence logique de la définition de l'identité comme résultat d'une interaction et non comme une définition substantiviste » (Taboada-Léonetti, 1990, p. 49). Ainsi, dans les deux approches, les comportements des individus en situation interculturelle sont issus des processus stratégiques résultant de leur élaboration individuelle et collective, et expriment, dans leur mouvance, les adaptations opérées, au jour le jour, en fonction de la variation des situations et des enjeux qu'elles suscitent. Cependant, le nombre de stratégies d'acculturation identifiées étant relativement réduit, il est nécessaire de les articuler avec les nombreuses nuances et détails introduits par Camilleri (Dasen et Ogay, 2000).

Les deux approches partagent également l'idée selon laquelle les stratégies d'acculturation et identitaires sont multidimensionnelles. On ne peut situer l'individu sur une seule dimension à mi-chemin entre les deux cultures en contact. En ce sens, le modèle d'acculturation de Berry $(1989,2000)$ se base sur le croisement de deux dimensions : la volonté d'avoir des contacts et des participations avec la société d'accueil et d'adopter ses valeurs, et la volonté de maintenir sa culture d'origine. S'il fallait faire le lien avec le modèle des stratégies identitaires de Camilleri (1989, 1990), la première correspond au pôle pragmatique de l'identité dont la fonction est adaptative. II s'agit de trouver une place psychologique et sociale admissible. La deuxième renvoie au pôle ontologique. La fonction est ici intégratrice, il faut préserver le moi en tant qu'unité intégrée. 
Malgré cette complémentarité des deux modèles tant au niveau de la notion de stratégie qu'au niveau de la notion d'identité dans une situation de contacts de cultures, ils se sont essentiellement développés en parallèle. II nous semble pourtant heuristique de proposer une articulation de ces deux approches, en précisant les stratégies identitaires correspondant à chaque stratégie d'acculturation, comme nous le proposons dans le tableau suivant (tableau 3).

Tableau 3 : Articulation des stratégies d'acculturation et des stratégies identitaires

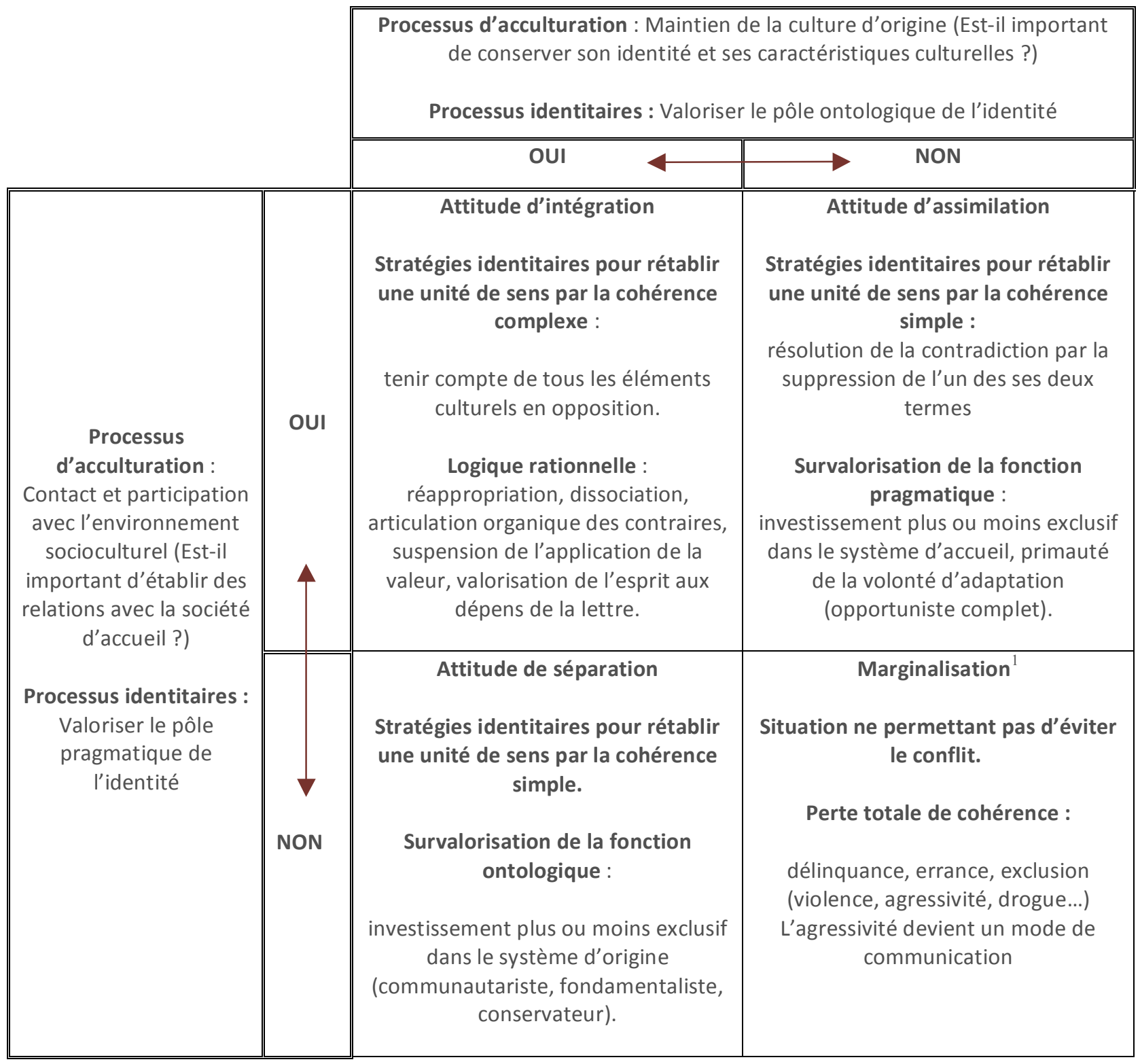

En effet, les stratégies d'acculturation telles qu'elles étaient proposées par Berry (1989, 1990, 2000) sont des attitudes qui renvoient aux préférences individuelles et à des positionnements d'« idéal type » dans la société (soit

\footnotetext{
${ }^{1}$ Nous ne pouvons pas considérer la marginalisation comme une stratégie ou une finalité, mais plutôt comme une situation pathologique comme le souligne Berry lui-même (2000). Cette situation n'a pas d'équivalant dans le modèle de Camilleri (1990). Il en est de même pour les stratégies de modération des conflits du modèle de Camilleri, qui n'ont pas d'équivalant dans le modèle des stratégies d'acculturation de Berry.
} 
la façon dont on aimerait vivre idéalement). Par ces positionnements, l'individu place l'unité de sens et la cohérence identitaire comme finalité. Pour atteindre ces deux objectifs, il met en place des stratégies identitaires conscientes ou non (ce sont des comportements) qui restent au niveau de la "réalité effective " (ce qu'on fait en réalité et comment s'y prendre pour vivre) (Camilleri, 1996a). Nous pouvons donc considérer les stratégies identitaires comme des moyens (comportements) mis en œuvre en vue de finalités (attitude d'assimilation, d'intégration et de séparation). Autrement dit, les stratégies identitaires illustrent les processus d'acculturation et permettent de saisir la dynamique de la restructuration identitaire à l'œuvre dans ces processus (Camilleri, 1990).

Partant d'une approche interactionniste de l'identité, les analyses des stratégies identitaires adoptées et des contacts de cultures doivent garder un certain déterminisme, tout en considérant l'individu comme singulier. II faut comprendre l'individualité comme issue de différentes influences environnementales et accorder à l'individu un caractère unique. Les stratégies identitaires peuvent alors correspondre à une création propre à chaque individu, répondant aux exigences sociales et psychologiques.

Cette articulation entre les deux modèles a comme objectif de stimuler la réflexion sur la manière d'intégrer les processus psychologiques individuels et I'influence sociale (des groupes, du contexte socio-politique), sur l'utilisation des stratégies d'acculturation des immigrants, notamment en termes de choix et d'impacts. Bien évidement, cette proposition ne prétend être ni exhaustive et exclusive, ni un modèle et les limites de cet article ne permettent pas de l'approfondir. Elle constitue néanmoins une ouverture pour toute recherche en psychologie interculturelle ayant comme objectif de mettre en évidence les caractères dynamiques et stratégiques de l'identité qui naissent de l'interaction sociale et de la relation dialectique entre le social et le personnel, entre l'individuel et le collectif dans une situation interculturelle.

Quant à la méthodologie, il serait nécessaire de mettre cette articulation à l'épreuve des faits en s'appuyant sur une approche qualitative et quantitative, privilégiant ainsi des méthodes mixtes qui prennent en considération le caractère dynamique de l'identité et le caractère composite des stratégies d'acculturation (Brégent, Mokounkolo et Pasquier, 2008).

\section{Conclusion}

En proposant une approche basée sur différents niveaux d'analyse et construite à partir du concept de l'identité dans ses multiples dimensions (personnelle et collective, attitudinale et comportementale, sociale et culturelle), nous avons cherché à mettre en avant toute la complexité de la psychologie interculturelle.

Malgré le risque de rendre difficile l'étude des contacts de cultures, cette multidimensionnalité, présentée dans le tableau 3, permet néanmoins de rendre compte de la complexité du phénomène en question. Ceci est indispensable au travail d'observation du chercheur, lui-même évoluant dans un contexte socio-politique et culturel donné, tout en étant lui aussi, à l'instar des populations migrantes qu'il étudie, en situation interculturelle (Derivois, 2007).

L'objectif de l'articulation des stratégies identitaires et des stratégies d'acculturation proposée dans cet article est d'une part de dépasser un cloisonnement scientifique et idéologique et, d'autre part, de nous extraire d'un " ethnocentrisme disciplinaire»(Clanet, 1990, p. 201), qui aboutit dans la plupart des cas à un processus de catégorisation et à un antagonisme entre un " nous » qui serait dans le vrai et un " eux " qui serait dans l'erreur. Cette catégorisation est le résultat des constructions historiques et culturelles des notions clés (culture, race, ethnie) de la recherche interculturelle (Troadec, 2001), notions qui ont longtemps à la fois nourri et affaibli la valeur conceptuelle de l'identité.

L'une des principales limites de cette réflexion théorique concerne le manque d'une analyse systématique des bases empiriques du modèle des stratégies identitaires et de celui des stratégies d'acculturation. Cette question incontournable aurait allongé considérablement ce document. Les travaux empiriques ayant validé chacun des modèles et la mise à l'épreuve de notre proposition d'articulation devront faire l'objet de recherches futures. 


\section{Références bibliographiques}

Amin, A. (2005). L'intégration des jeunes Français issus de l'immigration, le cas des jeunes issus de l'immigration arabo-musulmane et turque : Maghreb, Moyen-Orient et Turquie. Connexions : Différence Culturelles, Intégration et Laïcité, 83, 131-147.

Amin, A. (2007). Dynamique interculturelle et processus d'interculturation : Représentations sociales, identifications et sentiment d'exclusion. Le cas des jeunes Français issus de l'immigration maghrébine et turque (thèse de doctorat non publiée). Université Lyon 2, France.

Andronikof-Sanglade, A. et Franchi, V. (2001). Les modèles de l'identité de Camilleri, Markus et Berry : réflexion épistémologique. Dans M. Lahlou et G. Vinsonneau (dir.), La psychologie au regard des contacts de cultures (p. 55-64). Lyon : I'Interdisciplinaire.

Balkaïd, N. (2000). Processus d'interculturation et structuration identitaire. Catégorisation, formation des catégories et structure des appartenances : le cas des migrants marocains en France (thèse de doctorat non publiée). Université de Toulouse-Le Mirail, France.

Balkaïd, N. et Guerraoui, Z. (2003). La transmission culturelle, le regard de la psychologie interculturelle. Empan, 51, 124-128.

Berry, J. (1980). Acculturation as varieties of adaptation. Dans P. M. Padilla (dir.), Acculturation theory, models, and some new findings (p. 9-25). Boulder: Westview.

Berry, J. (1989). Acculturation et adaptation psychologique. Dans J. Retschitzki, M. Bossel-Lagos et P. Dasen (dir.), La recherche interculturelle (p. 135-145). Paris : L'Harmattan.

Berry, J. (1990). Psychology of accultration. Dans J. Bernan (dir.), Nerbraska symposium on motivation: crosscultural perspectives (p. 201-233). Lincoln : University of Nerbraska Press.

Berry, J. (2000). Acculturation et identité. Dans J. Costa-Lascoux, M. A. Hily et G. Vermès (dir.), Pluralité des cultures et dynamiques identitaires : Hommage à Carmel Camilleri (p. 81-94). Paris : L'Harmattan.

Berry, J., Phinney, J., Sam, D. et Vedder, P. (2006). Immigrant youth: Acculturation, identity, and adaptation. Applied Psychology: An International Review, 55(3), 303-332.

Berry, J. et Sabatier, C. (2010). Acculturation, discrimination, and adaptation among second generation immigrant youth in Montreal and Paris. International Journal of Intercultural Relations, 34, 191-207.

Berry, J. et Sam, D. (1997). Acculturation and adaptation. Dans J. W., Berry, M. H. Segall et Ç. Kagitçibasi (dir.), Handbook of cross-cultural psychology (vol. 3) (p. 291-326). Boston : Allyn \& Bacon.

Bourhis, R., Barrette, G., El-Geledi, S. et Schmidt, R. (2009). Acculturation orientations and social relations between immigrant and host community members in California. Journal of Cross-Cultural Psychology, 40, 443-467.

Bourhis, R. et Bougie, E. (1998). Le modèle d'acculturation interactif : Une étude exploratoire. Revue Québécoise de Psychologie, 19, 75-114.

Bourhis, R., Moise, L., Perreault, S. et Senecal, S. (1997). Towards an interactive acculturation model: A social psychological approach. International Journal of Psychology, 32, 369-386.

Bourhis, R., Personnaz, M., Barrette, G. et Personnaz, B. (2002). Étude sur les orientations d'acculturation de Maghrébins et de Français d'origine en région parisienne. Dans C. Sabatier., H. Malewska-Peyre et F. Tanon (dir.), Identité, acculturation et altérité (p. 123-147). Paris : L'Harmattan.

Brégent, M., Mokounkolo, R. et Pasquier, D. (2008) Recherche et classification d'indicateurs d'acculturation à partir du contexte francophone. Psychologie Française, 53, 51-69.

Camilleri, C. (1989). La culture et l'identité : Champ notionnel et devenir. Dans C. Camilleri et M. Cohen-Emerique (dir.), Choc de cultures. Concepts et enjeux pratiques de l'interculturel (p. 21-73). Paris : L'Harmattan.

Alterstice - Revue Internationale de la Recherche Interculturelle, vol. $2, n^{\circ} 2$ 
Camilleri, C. (1990). Identité et gestion de la disparité culturelle : Essai d'une typologie. Dans C. Camilleri, J. Kastersztein, E. Lipianski, H. Malewska-Peyre, I. Taboada-Léonetti et A. Vasquez (dir.), Stratégies identitaires (p. 85-110). Paris : P U F.

Camilleri, C. (1992). Cultures et stratégies, ou les mille manières de s'adapter. Dans C. Halpern et J. C. RuanoBorbalan (dir.), Identité(s) : L'individu, le groupe, la société (p. 85-90). Auxerre : Sciences Humaines.

Camilleri, C. (1996a). Le champ et les concepts de la psychologie culturelle. Dans C. Camilleri et G. Vinsonneau (dir.), Psychologie et culture : Concepts et méthode (p. 9-80). Paris : Armand Colin.

Camilleri, C. (1996b). La psychologie interculturelle. Dans C. Tapia (dir.), Introduction à la psychologie sociale (p. 91119). Paris : Les Éd. d’Organisation.

Clanet, C. (1990). L'interculturel, introduction aux approches interculturelles en éducation et en Sciences Humaines. Toulouse : Presses Universitaires du Mirail.

Dasen, P. (2001). Intégration, assimilation et stress acculturatif. Dans C. Perregaux, T. Ogay, Y. Leanza et P. Dasen (dir.), Intégrations et migrations : Regards pluridisciplinaires (p. 188-210). Paris : L'Harmattan.

Dasen, P. et Ogay, T. (2000). Pertinence d'une approche comparative pour la théorie des stratégies identitaires. Dans J. Costa-Lascoux, M. A. Hily et G. Vermès (dir.), Pluralité des cultures et dynamiques identitaires : Hommage à Carmel Camilleri (p. 55-80). Paris : L'Harmattan.

Denoux, P. (1994). Vers une nouvelle définition de l’interculturation. Dans J. Blomart et B. Krewer (dir.), Perspectives de l'interculturel (p. 67-81). Paris : École Norm. Sup. de St-Cloud/L'Harmattan.

Denoux, P. (1994-1995). L’identité interculturelle. Bulletin de psychologie, 48(419), 264-270.

Derivois, D. (2007). Interculturalités et relations drogues-crimes à l'adolescence. Enjeux épistémologiques et méthodologiques. Revue Internationale de Criminologie et de Police Technique et Scientifique, avril-juin, 215225.

Guerraoui, Z. (1992). Interculturation et contraception, étude comparative entre deux populations d'origine maghrébine en France : Femmes migrantes et filles de migrants (thèse de Doctorat non publiée). Université de Toulouse-Le Mirail, France.

Guimond, S. (2010). Psychologie sociale : Perspective multiculturelle. Belgique : Mardaga.

Kanouté, F. (2002). Profils d'acculturation d'élèves issus de l'immigration récente à Montréal. Revue des sciences de l'éducation, 28(1), 171-190.

Lipiansky, E., Taboada-Léonetti, I. et Vasquez, A. (1990). Introduction à la problématique de l'identité. Dans C. Camilleri., J. Kastersztein, E. M. Lipianski, H. Malewska-Peyre, I. Taboada-Léonetti et A. Vasquez (dir.), Stratégies identitaires (p. 7-26). Paris : Presses universitaires de France.

Malewsk-Peyre, H. (1989). Problèmes d'identité des adolescents enfants de migrants et travail social. Dans C. Camilleri et M. Cohen-Emerique (dir.), Chocs de cultures : concepts et enjeux pratiques de l'interculturel (p. 117-134). Paris : L'Harmattan.

Malewska-Peyre, H. (1990). Le processus de dévalorisation de l'identité et les stratégies identitaires. Dans C. Camilleri, J. Kastersztein, E.-M. Lipianski, I. Taboada-Léonetti et A. Vasquez (dir.), Stratégies identitaires (p. 111-141). Paris : Presses universitaires de France.

Manço, A. (2006). Processus identitaires et intégration. Approche psychosociale de l'intégration. Paris : L'Harmattan.

Redfield, R., Linton, R. et Herskovits, M. (1936). Memorandum on the study of acculturation. American Anthropologist, 38(1), 149-192.

Sabatier, C. (2001). Les études sur l'acculturation sont elles universelles ou contextualisées? Perspective française. Bulletin de I'ARIC, 36, 1-5. 
Sabatier, C. et Berry, J. (1994). Immigration et acculturation. Dans R. Y. Bourhis et J. Ph. Leyens (dir.), Stéréotypes, discrimination et relation intergroupes (p. 261-291). Belgique : Mardaga.

Sabatier, C. et Boutry, V. (2006). Acculturation in francophone European societies. Dans D. L. Sam et J. W. Berry (dir.), The Cambridge handbook of acculturation psychology (p. 349-367). New-York (NY) : Cambridge University Press.

Taboada-Léonetti, I. (1990). Stratégies identitaires et minorités : Le point de vue du sociologue. Dans C. Camilleri, J. Kastersztein, E. Lipianski, I. Taboada-Léonetti et A. Vasquez (dir.), Stratégies identitaires (p. 43-83). Paris : Presses universitaires de France.

Tajfel, H. et Turner, J. (1979). An integrative theory of intergroup conflict. Dans W.G. Austin et S. Worchel (dir.), The social psychology of intergroup relations (p. 33-48). Monterey : Books/Cole.

Tajfel, H. et Turner, J. (1986). The social identity theory of intergroup behaviour. Dans S. Worchel et W. Austin (dir.), Psychology of Intergroup Relations (p. 7-24). Chicago: MI Nelson-Hall.

Troadec, B. (2001, 24-28 sept.). Approche culturelle et interculturelle comparative : Vers une intégration de paradigmes complémentaires. Communication présentée au VIII ${ }^{\mathrm{C}}$ Congrès de l'Association pour la Recherche Inter-Culturelle (ARIC), Recherche et pratique interculturelles : nouveaux espaces, nouvelles complexités?, Genève, Suisse.

Vasquez, A. (1984). Les implications idéologiques du concept d'acculturation. Les Cahiers de sociologie économique et culturelle, 1, 83-121. 